\title{
Fault Tolerant System for Sparse Traffic Grooming in Optical WDM Mesh Networks Using Combiner Queue
}

\author{
Sandip R. Shinde \\ Research Scholar, Sathyabama University, Chennai \& \\ Assistant Professor, Vishwakarma Institute of Technology, \\ Pune, India \\ Dr. Suhas H. Patil \\ Professor, Dept of Computer Engineering, College of \\ Engineering, Bharati Vidyapeeth University, \\ Pune, India
}

\author{
Dr. S. Emalda Roslin \\ Associate Professor, Department of Electronics \& \\ Communication Engineering, Sathyabama University, \\ Chennai, India
}

\author{
Archana S. Shinde \\ Assistant Professor, Department of Information Technology, \\ Sinhagad Acedamy Of Engineering, \\ Pune, India
}

\begin{abstract}
Queuing theory is an important concept in current internet technology. As the requirement of bandwidth goes on increasing it is necessary to use optical communication for transfer of data. Optical communication at backbone network requires various devices for traffic grooming. The cost of these devices is very high which leads to increase in the cost of network. One of the solutions to this problem is to have sparse traffic grooming in optical WDM mesh network. Sparse traffic grooming allows only few nodes in the network as grooming node (G-node). These G-nodes has the grooming capability and other nodes are simple nodes where traffic grooming is not possible. The grooming nodes are the special nodes which has high cost. The possibility of faults at such a node, or link failure is high. Resolving such faults and providing efficient network is very important. So we have importance of such survivable sparse traffic grooming network.

Queuing theory helps to improve the result of network and groom the traffic in the network. The paper focuses on the improvement in performance of the backbone network and reduction in blocking probability. To achieve the goals of the work we have simulated the model. The main contribution is to use survivability on the sparse grooming network and use of combiner queues at each node. It has observed that Combiner queuing alone does the job of minimizing blocking probability and balancing the load over the network. The model is not only cost effective but also increases the performance of network and minimizes the call blocking probability.
\end{abstract}

Keywords-optical communication; sparse traffic grooming; survivability; fault tolerance; Combiner Queue; WDM

\section{INTRODUCTION}

Due to increasing demand of bandwidth it is very important for service providers to provide efficient and cost effective solutions to fulfill the need of society. Bandwidth requirements are fulfilled by the use of optical fibers. Optical fibers are providing terabytes of bandwidth. The signal attenuation and distortion is low and also there are very less power requirements. Due to these factors optical network is widely used in the data network. WDM (Wavelength Division
Multiplexing) is used to fulfill the requirement of users over optical network. The main idea of optical WDM network is that, maximum number of users can use network for the transfer of data. Our network must have sufficient bandwidth to fulfill the requirement of bandwidth hungry society. Concurrency will be provided in transmission among the various users in optical communication. WDM divides the bandwidth into multiple frequencies or channels. These are non-overlapping wavelength channels. It is very important to provide support for low traffic rate than the available capacity of wavelength. A single optical channel of STS-1 (51.84Mbps) may be used for that purpose which provides support up to wavelength capacity.

It is very important to transfer low speed data traffic on high-speed networks. Traffic grooming will transfer this low speed traffic to high-speed. The capacity of each wavelength is OC- $n$ it means $n * 51.84 \mathrm{Mbps}$. In traffic grooming you have to establish a light path to fulfill the requirement of the end user. The light path is established from source to destination from very low capacity to high capacity up to the maximum capacity of the channel. This traffic grooming problem is subcategories into four problems

- Design of virtual topology with light paths

- Routing the light paths over the physical topology

- Assignment of wavelengths to light paths

- Traffic routing on the virtual topology

For providing these services at each node we need some kind of hardware and software so that the nodes will have grooming capability. The ADM (Add/Drop Multiplexers), Optical ADM (OADM), Optical Cross Connects (OXC), etc which are required for grooming. Due to the requirement of these devices and additional software's at each node the cost of network goes on increasing. The sparse grooming is a technique in which few nodes will be equipped with grooming capabilities and few nodes without grooming capabilities. Due 
to reduction in the number of nodes of grooming capability cost of network will be reduced. But reducing the cost is not the only objective of the research community. The main target is to get the good or acceptable performance in minimum cost so that affordable solutions and services will be provided to the end user.

In the backbone network when the grooming is provided it may result into the cost effective best performance network. But sometimes due to some reasons different types of faults may occur and the performance degrades. Faults may be due to failure of nodes or failure of links. Therefore, it is very important to provide survivability to sparse grooming in optical WDM mesh network. Survivability is provided to the network so that it becomes fault tolerant system.

Main contribution of this paper is fault tolerant system for sparse grooming in optical WDM mesh network using combiner queuing. Work focuses in this paper is on node failure in the optical WDM mesh network.

Rest of the paper is organized in sections and it follows as in section II we discuss literature survey, section III focused on proposed methodology, section IV deals with implementation architecture and performance of proposed methodology. Conclusions are given in section $\mathrm{V}$ and section VI focuses on future scope.

\section{LITERATURE REVIEW}

Backbone network has moved from ring to mesh topology. Traffic grooming problem is addressed in both ring and mesh topology. The current focus of traffic grooming is on mesh topology [1-8]. Two approaches of traffic grooming are static and dynamic which are addresses by many papers [9-13]. Heuristics for Sparse Traffic Grooming in Dynamic WDM Optical Mesh Networks is proposed by S. R. Shinde et. al. [14] heuristic algorithms are proposed for G-node selection. On the selected G-node grooming will be performed which results in efficient solution. The focus was also the load balancing on the routes in the optical network. Where as in [15] Modified Multiobjective Meta-heuristics is proposed for Sparse Traffic Grooming in Optical WDM Mesh Networks. Any of these works is not addressing the problem of survivability over the WDM optical Mesh network.

Survivability in optical network is addressed in [16] and the focus of the work is classification of disaster survivability in optical networks. Authors have classified the survivability on the basis of present work and they have focused on survey of that classification. They also classified disasters and addressed impact of classification on communication networks. Failures may be anything of node failure, link failure, channel failure, or software failure. To recover from fault there are two solutions one is protection and other is restoration [17].

In protection method dedicated resources are required. For restoring the services affected by failure in restoration the emergency capacity available within the network is utilized. Protection method is either path protection or link protection. In path protection alternate route is reserved in advance for the source and destination connection when a connection is set up. The path protection is again either dedicated or shared path protection. In dedicated path the alternate route is only for the one route. And in shared path protection the alternate route is shared as a backup route for more than one path as failure may not occur continuously.

As in the case of path protection link protection is another situation. In link protection the link is protected as alternate link to one where connection is established. This link protection is again dedicated and shared link similar to path protection. In restoration method there are also path restoration and link restoration. In path restoration after failure the system has to find out alternate path and for that the path will be searched at run time. This is not good method for high priority data. In link restoration as well the link will be made available by search after the failure. This is slow method and loss of data may possible.

Focus of this paper is to provide better solution for the various problems of the current system. In the literature it is observed that focus of research is either sparse traffic grooming or survivability on WDM optical network. This work has the bidirectional focus. First of all the system is made sparse traffic grooming for Poison traffic and load balancing. Upon successful sparse traffic grooming the vulnerabilities in optical network are considered and system is provided with the better solution as a fault tolerant system over sparse traffic grooming.

\section{SURVIVABLE SPARSE TRAFFIC GROOMING USING COMBINER QUEUES}

Traffic grooming is the technique in which all nodes have the capability of wavelength conversion it means all nodes are grooming node (G-node). The performance of full traffic grooming network is good but the network is not cost effective. If the cost of the network is very high, then the solutions are not affordable to the end users. So it is very important to provide cost effective best solutions to the society. One such a network is sparse traffic grooming optical WDM mesh network.

In sparse traffic grooming optical WDM mesh network only some nodes has the grooming capability. Only grooming nodes perform wavelength conversion whereas the nongrooming node does not have the capability of wavelength conversion. The nodes which have grooming capability are highly important nodes in the sparse traffic grooming. So these G-nodes are placed in such a way that less number of grooming nodes must give acceptable performance which is nearly equal to full grooming. G-nodes are selected based on various factors such as nodal degree, maximum traffic node, any random $\mathrm{m}$ nodes, etc. But any of these methods does not give the best solutions we are using the heuristic in [14] by applying combiner Queue to the nodes.

At each node a combiner queue will be applied and each node has its own initial weight. This weight is going to be increased based on the factors of degree and traffic at that node.

A. Algorithm : G-Node Selection with Combine Queиe (CQ)

1) Assign the weights to each node.

2) Find the Nodal degree of given Node.

3) Increment the weights by adding the nodal degree to each node. 
4) Generate the Poisson traffic in the network.

5) Count the number of requests in each queue and add that to the weight of the nodes.

6) If the CQ is full, that node will be declared as G-Node.

7) Else the nodes with highest weights are declared as $G$ nodes.

Once the G-nodes are decided for the 14 node NSF net topology shown in fig. 1 then data traffic will be generated dynamically with the Poisson distribution.

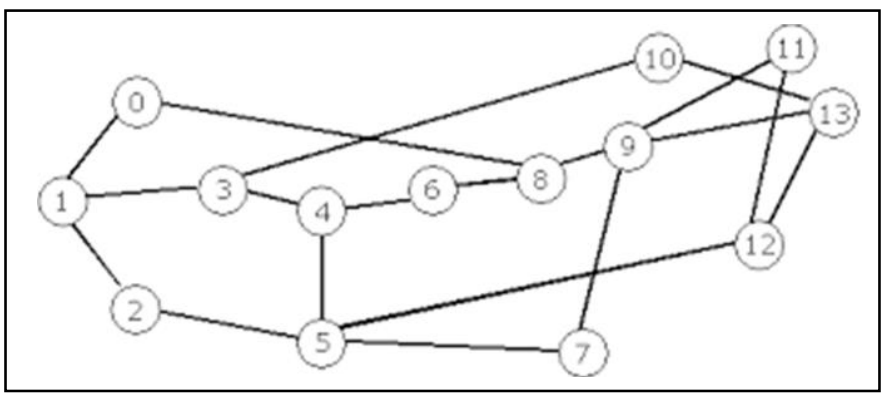

Fig. 1. 14-node NSF NET topology

System is modeled as the Markov chain model where each node is represented as one state. Rate of arrival is $\lambda$ at each link and the service provided by the link is given by $\mu$. Utilization ( $\rho$ ) of the channel is given by the rate of arrival by the service rate. Due to combiner queue there are some queues at each node which are not failure say $i$ and there are $j$ queues where ongoing calls are there. Therefore $\rho=\lambda / m \mu$ and the number of requests which are blocked will be given by the call blocking probability $\mathrm{P}[$ block $]$ is

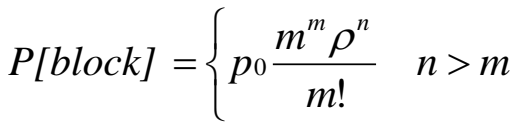

Where is initial probability, $n$ is the number of calls and $m$ is the number of channels at that node. Here all requested are arrived at one the queue from CQ. All these request needs are analyzed and appropriate channel is assigned. If the channel is not available then the request is in wait state and the CQ will calculate the time required to get the service from that queue and other queues at that node. Then the request will be requeued in the efficient queue of the same node. If the node is grooming node then wavelength conversion take place and probability of service in time will get increased.

If the failure of the path/link occurs then the other path/link is protected for the same. Only change here which is very important in terms of full grooming and sparse grooming is that the path/link provided as an alternate to the one selected must have nearest G-node on the path or link. Shortest path routing algorithm is used to find shortest path. The wavelength assignment algorithm as given is applied to assign the appropriate wavelength.

\section{B. Wavelength assignment algorithm:}

1) Check the Request Ri in each $C Q$,

2) if Wi available and fulfill Ri with available wavelength, assign to it.
3) Else if node is G-node use wavelength conversion and assign the channel to that request $R i$

4) Analyze the waiting time at each Queue of $C Q$ at that node

5) Divert that request to the queue where waiting time is less of the same node and check for Wi

6) If the waiting time is too high in all queues block that call.

\section{IMPLEMENTATION ARCHITECTURE AND PERFORMANCE}

To evaluate our proposed methodology we have taken a network with arbitrary mesh topology. We have conducted simulation experiments on 14-node NSFNET topology with OC-192. The source and destination for the request are generated exponentially with Poisson distribution. At each node we have design Combiner Queue for each physical link.

The simulation environment is architecturally decomposed into different modules. This simulation has build in Omnet++ simulator. The node design consists of Router for routing, App module for generating source destination request and Combiner Queues.

Fig. 2 shows a Node Architecture. Queues are design to handle the requests which are arriving at the node. Router routes the request according to the shortest path to the specified destination. "App" design is used to generate the request in exponential order where it is sent to destination by router.

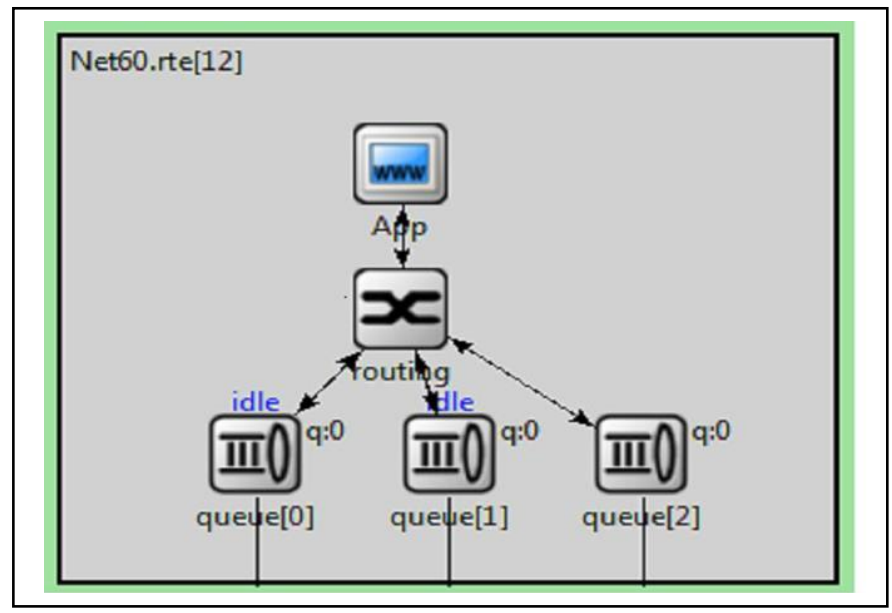

Fig. 2. Node Architecture of each node

The performance of proposed algorithm with existing few algorithms like CLPGA, MTT have been compare with parameters like Blocking probability, Network cost with Traffic Load. The proposed algorithm and MTT(Maximum Total traffic) are compared for number of sparse node selected for varying time as the traffic changes. This gives idea about how many nodes will be sparse node in the network. Based on this we have finalized number of sparse node and varying results for various parameters are achieved.

The graph for number of Sparse node selected for particular ongoing time has been plotted shown in Fig. 3 


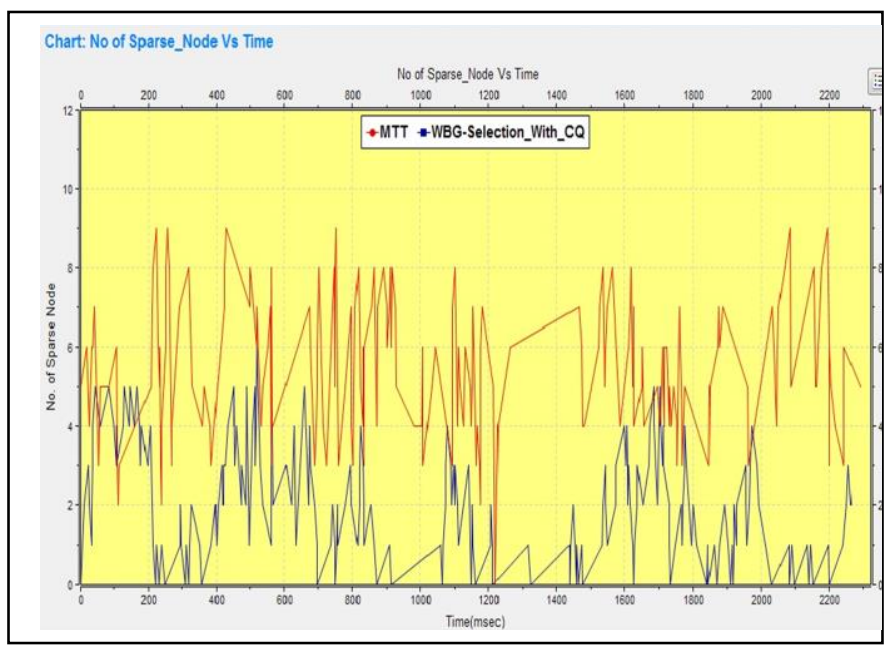

Fig. 3. No.of Sparse Node Vs Time in msec

The various algorithms are compared for the blocking probability with varying load and it is observed that the proposed method using combiner Queue gives good results as shown in Fig. 4.

Here BBORM (Bypass Based Optical Routing Algorithm) which make use of Buffering concept is compared with proposed algorithm and also the MTT has been considered over here.

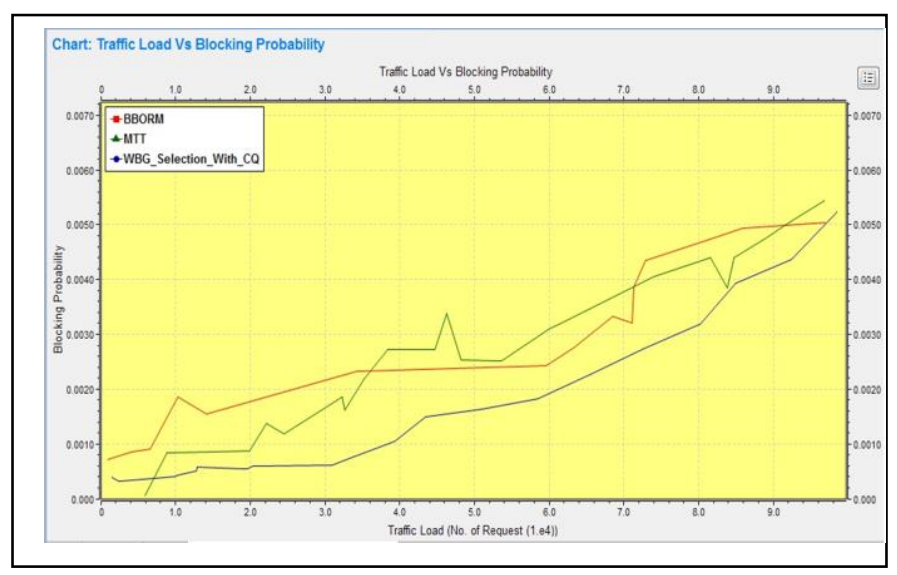

Fig. 4. Blocking probability Vs Traffic Load

Main goal of the proposed method is to reduce the network cost, and provide survivability as a fault tolerant system is achieved and is shown in fig.5.

We have seen as the load increases in the network the performance of the network degrades but in the proposed methodology the performance degradation is negligible compare to some existing methods to which we compared our results which is shown in fig.6.

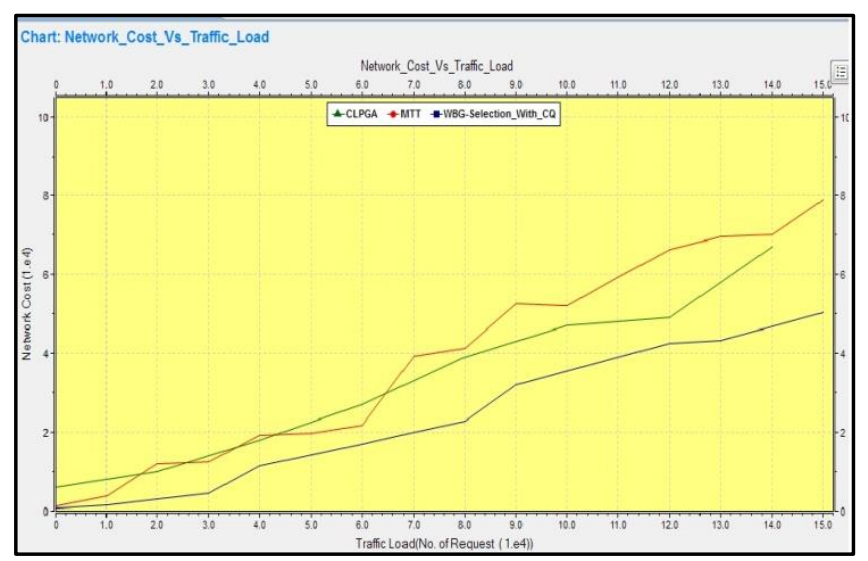

Fig. 5. Network cost Vs Traffic load

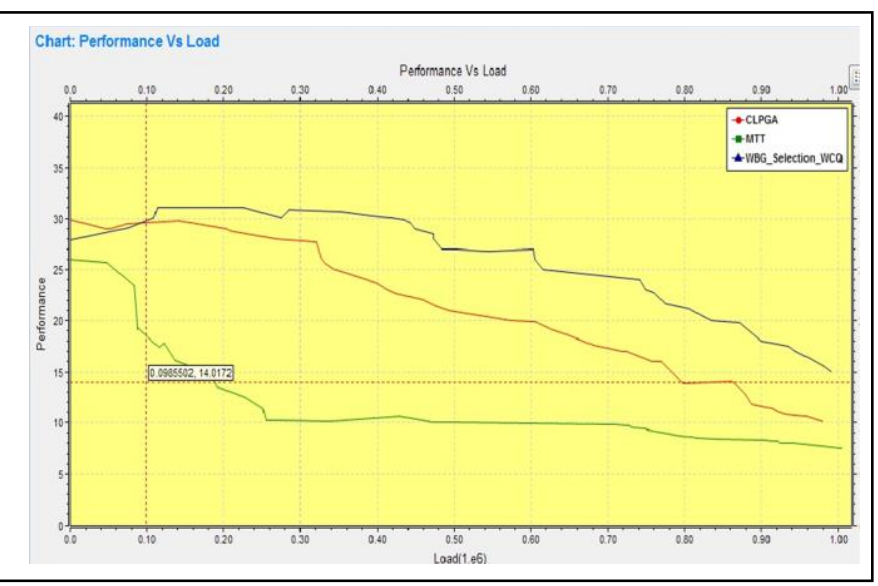

Fig. 6. Performance Vs Load

We also tried by varying the number of sparse node i.e. Gnode and with varying load and we observed that blocking probability in the proposed method in any situation for any number of G-nodes is less than the available methods as shown in fig. 7.

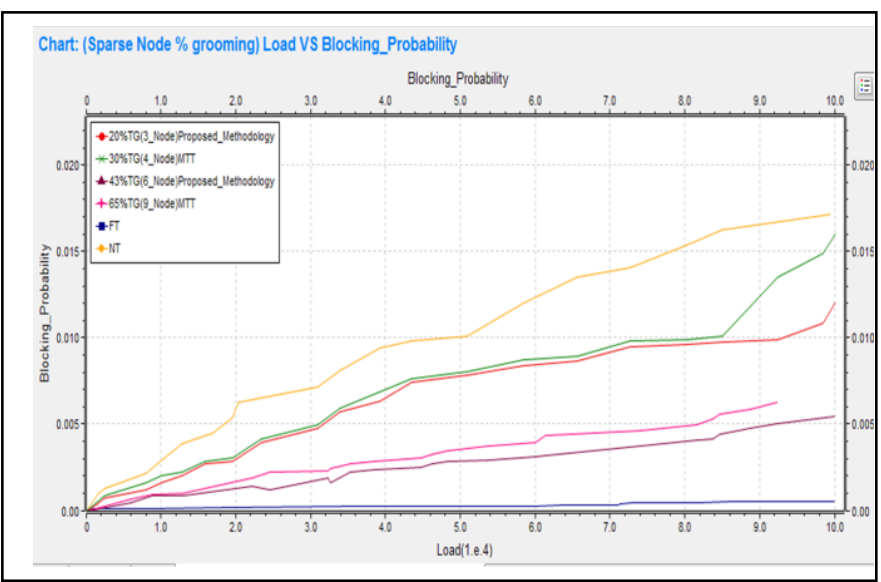

Fig. 7. Blocking Probability for Varying Sparse Node Vs Load 


\section{CONCLUSIONS}

Experimental work carried out using OMNet++ for fault tolerant system for sparse traffic grooming is modeled using combiner queue gives better performance by reducing the cost of network. The system is fully robust for the vulnerabilities at node failure. For fulfilling the demands of several customers over optical network Bandwidth distribution is one of the most promising ways to improve the network utilization.

The proposed methodology minimizes the total cost of traffic grooming and wavelength conversion equipment used in WDM networks with sparse traffic grooming and wavelength conversion resource without hindering the network blocking performance. We have compared our methodology with existing methodologies and from results it is concluded that our methodology is cost effective and also have less blocking probability. The GRWA problem is solved in most efficient manner in our methodology.

From results it is concluded that proposed methodology is more cost efficient and also has less blocking probability which is our objectives. Also, Load balancing over the network has been achieved by our combiner queuing model which is our one of the objective.

Our wavelength assignment algorithm strives to avoid wavelength conversion and wavelength bandwidth fragmentation by using queuing model where it is sent over respective paths by maintaining wavelength continuity constraint. The strength of the methodology lies from their applicability, minimizing the total cost of traffic grooming and wavelength conversion equipment used in WDM networks without hindering the network blocking performance.

\section{FUTURE SCOPE}

Energy consumption for traffic grooming is the future area of research which needs to be focus in order to minimize the energy requirement of network. This is also indirectly talks about the cost of the network.

Also, the power consumption for the grooming resources and minimizing the complexity of resource allocation is one of major area of research which is coming in Optical traffic grooming.

\section{REFERENCES}

[1] K. Zhu and B. Mukherjee, Traffic grooming in an optical WDM mesh network IEEE J. Selected Areas Commun. 20 (1) (2002) 122-133.

[2] K. Zhu and B. Mukherjee, A review of traffic grooming in WDM optical networks: Architectures and challenges, SPIE Opt. Networks Mag. 4 (2) (2003) 55-64.

[3] E. Modiano and P. J. Lin, Traffic grooming in WDM networks, IEEE Commun. Mag. 39 (2001) 124-129.

[4] J. Q. Hu and B. Leida, Traffic grooming, routing and wavelength assignment in optical WDM mesh networks, Proc. of IEEE INFOCOM (2004) 495-501.

[5] R. Dutta, G. N. Rouskas, On optimal traffic grooming in WDM Rings, IEEE J. Selected Areas Commun. 20 (1) (2002) 110-121.

[6] L. Chiu and E. H. Modiano, Traffic grooming in algorithms for reducing electronic multiplexing costs in WDM ring networks, IEEE/OSA J. Lightw. Technol. 18 (1) (2000) 2-12.

[7] J. Wang, V. R. Vemuri, W. Cho, and B. Mukherjee, Improved approaches for cost effective traffic grooming in WDM ring networks: ILP formulations and single-hop and multi-hop connections, IEEE/OSA J. Lightw. Technol. 19 (11) (2001) 1654-1653.

[8] X. Zhang and C. Qiao, An effective and comprehensive approach for traffic grooming and wavelength assignment in SONET/WDM rings, IEEE/ACM Trans Networking 8 (5) (2000) 608-617.

[9] C. Xin, Blocking analysis of dynamic traffic grooming in mesh WDM optical networks, IEEE/ACM Trans Networking 15 (3) (2007) 721-733.

[10] C. Xin, Dynamic traffic grooming in optical networks with wavelength conversion, IEEE J. Selected Areas Commun. 25 (9) (2007) 50-57.

[11] O. Awwad, A. I. Al-Fuqaha, A. Rayes, Traffic grooming, routing and wavelength assignment in WDM transport networks with sparse grooming resources, Comput. Commun. 30 (2007) 3508-3524.

[12] C. Xin, B. Wang, X. Cao, J. Li, Logical topology design for dynamic traffic grooming in WDM mesh networks, J. Lightw. Technol. 24 (2006) 2267-2275.

[13] K. Roy and M. K. Naskar, Simulation-based SONET ADM optimization approach for dynamic traffic grooming in WDM optical networks, Photon Netw. Commun. 16 (2008) 11-21.

[14] S. R. Shinde, S. H. Patil, Heuristics for Sparse Traffic Grooming in Dynamic WDM Optical Mesh Networks, IEEE International Conference on Computing Communication Control and Automation (ICCUBEA), (2015) page- 159 - 163

[15] Sandip Shinde, Dr. S.H. Patil , Manasi Gulwani, Modified Multiobjective Metaheuristics for Sparse Traffic Grooming in Optical WDM Mesh Networks, Elsevier Procedia Computer Science 57 ( 2015 ) $980-987$.

[16] M. Farhan Habiba, Massimo Tornatore, Ferhat Dikbiyik, Biswanath Mukherjee, Disaster survivability in optical communication networks, Computer Communications, Volume 36, Issue 6,( 2013), Pages 630644.

[17] B. Mukherjee, “Optical WDM Networks”, Springer Publication, 2006. 